\title{
Student perspective of GTA strategies to reduce feelings of anxiousness with cold-calling
}

\author{
Constance M. Doty ${ }^{1}$, \\ ${ }^{1}$ Department of Physics, University of Central Florida, 4111 Libra Drive, Orlando, FL, 32816 \\ Ashley A. Geraets ${ }^{2}$, Tong Wan ${ }^{1}$, Erin K. H. Saitta ${ }^{2}$ and Jacquelyn J. Chini ${ }^{1}$ \\ ${ }^{1}$ Department of Physics, University of Central Florida, 4111 Libra Drive, Orlando, FL, 32816 \\ ${ }^{2}$ Department of Chemistry, University of Central Florida, 4111 Libra Drive, Orlando, FL, 32816
}

\begin{abstract}
We investigated student perceptions of cold calling on their feelings of anxiousness and how graduate teaching assistants (GTAs) alleviated these feelings when students shared their ideas publicly in the context of tutorial and laboratory sessions. Physics and chemistry GTAs who led active-learning tutorials and labs practiced cold calling paired with error framing with avatar-students in a mixed-reality simulator at the beginning of the semester. Then, we observed the GTAs teaching real students in their actual classroom. We recruited eleven students from sections led by GTAs who were observed to use cold calling in their classroom to participate in semi-structured interviews. Several students reported that cold calling increased their feelings of anxiousness. However, students also reported that GTAs used strategies paired with cold calling that reduced their feelings of anxiousness, such as acknowledging student responses as valuable and remembering student names. We discuss implications for professional development on active learning strategies.
\end{abstract}

2019 PERC Proceedings edited by Cao, Wolf, and Bennett; Peer-reviewed, doi.org/10.1119/perc.2019.pr.Doty Published by the American Association of Physics Teachers under a Creative Commons Attribution 4.0 license. Further distribution must maintain attribution to the article's authors, cover page, and DOI. 


\section{INTRODUCTION}

The STEM education community has used a variety of training formats to support graduate teaching assistants' (GTA) development of the pedagogical skills needed for active learning, such as pedagogy seminars and practice microteaching to peers [1-4]. In addition, researchers and educators have described a variety of pedagogical strategies instructors can use to support student engagement in active learning (e.g., [6-7]). Two instructional strategies that promote student participation in the classroom are cold calling and error framing.

In cold calling, an instructor calls on a non-volunteering student by name to respond to a question (the instructor may or may not have prompted the students to have a response ready before asking them to answer) [1,7]. Cold calling has been reported to increase student participation with active learning activities like whole class discussion [8]. Random call, a specific version of cold calling where the instructor uses a randomized list to select who to call on, has been demonstrated to increase equity in participation [9].

However, researchers have reported that cold calling can also increase students' feelings of anxiousness $[10,11]$. Cooper, Downing and Brownell propose students' fear of negative evaluation as a possible mechanism through which cold calling can increase anxiety [11]. In a classroom setting, fear of negative evaluation is students' fear of being negatively judged by their instructor or their peers when they participate in a classroom activity or share their ideas [12]. To reduce the potential negative impact of cold calling on students' affect, an instructor could pair cold calling with another teaching strategy to promote student engagement while also helping students feel comfortable with sharing their ideas in front of the class.

In error framing, the instructor creates an environment that error is framed as a natural part of the learning process [1,7,13-15]. Instructors can error frame while introducing an activity (e.g., by stating that students should expect to engage in "productive failure") [16] and in response to student errors (e.g., by framing student ideas as common and reasonable) [1]. Becker et al. and Eddy, Converse, and Wenderoth have suggested the use of error framing to decrease student anxiety related to active learning classroom activities and to encourage student participation in class [1,13]. However, in the same studies, instructors were rarely observed to use error framing $[1,13]$. Therefore, the impact of error framing on student affect is a continuing investigation.

In our study, we are engaged in a project exploring the use of a mixed-reality classroom simulator as a safe environment for STEM GTAs to practice instructional strategies with avatar-students. The simulator is mixedreality because GTAs are in a physical classroom while they interact with a virtual class of students who are puppeteered through human-in-the-loop technology. While GTA professional development often focuses on changing GTA beliefs about teaching and learning, it is also important to explore changes in GTAs' teaching actions and impacts on their students [5]. In this paper, we explore the impact of GTA use of cold calling on the undergraduate students they taught. We recruited students from the classes of GTAs who were observed to use cold calling and asked them about the strategies their GTAs used along with cold calling to increase student comfort. We identified seven strategies the undergraduate students reported their GTAs used that increased their comfort sharing their ideas in front of peers: 1) error framing; 2) allowing other students to assist with answering the cold call question; 3) building off of what students know; 4) providing neutral responses with explanations; 5) using student names; 6) calling on student groups; and 7) providing thinking time before cold calling. Based on these findings, we suggest that GTAs already have and can learn new strategies to reduce student anxiety associated with cold calling.

\section{METHODS}

\section{A. Context}

GTAs leading the introductory inquiry-based general chemistry lab [17] ( 24 students) and the introductory physics "mini-studio" (recitation and lab combined) [18] ( 32 students) participated in a one-day boot camp during the first week of the spring 2019 semester in addition to their weekly prep meetings. Each GTA participated in two sevenminute sessions in a mixed-reality simulator [19]. GTAs were prompted to practice cold calling and error framing with avatar-students while facilitating a whole class discussion. The GTAs rehearsed in the simulator in groups of two or three. Each GTA led one seven-minute session, followed by five minutes of feedback from the facilitators and a second round of seven-minute sessions with the avatarstudents. Throughout the spring 2019 semester, we observed ten GTAs in physics and eleven GTAs in chemistry teaching in their classrooms four times using a modified version of the Laboratory Observation Protocol for Undergraduate STEM (LOPUS) [20]. We added codes to the protocol for cold calling and error framing. We observed four GTAs (three in chemistry and one in physics) using cold calling and error framing in at least two of the four observations.

\section{B. Interviews}

We recruited eleven students from sections taught by the four identified GTAs via a survey posted on their course website to participate in one-hour semi-structured interviews about their experiences in their physics mini-studio or chemistry lab. Considering that students may have different class schedules and various comfort levels for individual interviews, students were allowed to choose either focus group or individual interviews. Seven students participated 
in individual interviews, and four students participated in two-person focus groups; the students in each focus group shared the same GTA. Due to the social nature of focus groups, participants' ideas voiced during a focus group may be different than the ideas they would provide during an individual interview. Thus, we present our findings disaggregated by interview type in Table 1. All interviews were audio-recorded and transcribed.

Two researchers (C.M.D. and A.A.G.) conducted the semi-structured interviews. The interview protocol was developed after reviewing relevant literature (e.g., $[1,7,11]$ ) which provides evidence for the validity of our interpretations of what students say because the participants were prompted to talk about the relevant concepts. During the interviews, students were asked to discuss the impact of cold calling on their feelings of anxiousness [21] and were prompted to describe what their GTA has done to make them feel comfortable with sharing their ideas with the whole class or their lab group.

\section{Data analysis}

C.M.D. and A.A.G analyzed transcripts of the student interviews in multiple rounds using the constant comparative method to develop a codebook [22]. First, each researcher individually identified key phrases and documented emergent themes in the transcripts. We then compared phrases and themes to develop a codebook with seven codes for identified strategies, including code names, descriptions, and exemplar student quotes. Lastly, we each independently implemented the codebook on all the transcripts. We investigated inter-rater reliability (IRR) for our implementation of the seven codes using Gwet's AC1, which is robust to low trait prevalence [23]. Our Gwet's AC1 values ranged from 0.89 to 0.94 for all seven codes across the nine transcripts. Gwet's values greater than 0.81 indicate near perfect agreement; thus, we have evidence for the reliability of our implementation of the seven codes [24]. After the individual coding, we discussed discrepancies in our implementation of the codebook until we reached agreement. We present the agreed upon coding.

\section{Findings}

We found ten out of eleven students reported an increase in anxiety associated with their GTA's use of cold calling, comparable to Cooper, Downing and Brownell's findings from students in a large enrollment biology course [11]. However, we also found all the students had perceived their anxiety associated with cold calling to decrease when their GTA paired cold calling with another teaching strategy. We identified seven teaching strategies students reported their GTA to use with cold calling that increased their comfort with sharing their ideas in front of their peers, as shown in Table I. Since GTAs were instructed to rehearse error framing, we discuss this strategy first.
TABLE I. Teaching strategies students identified to reduce their feelings of anxiousness related to cold call. We indicate the type of interview: individual (I) or focus group (F). P (physics) and $\mathrm{C}$ (chemistry) indicate the discipline of the GTA. The number next to $\mathrm{P}$ or $\mathrm{C}$ indicates a different GTA in the same discipline. The number of participants for each GTA is shown.

\begin{tabular}{cccccccc}
\hline \hline Strategy & $\begin{array}{c}\text { P1: } \\
\mathrm{I}=1\end{array}$ & $\begin{array}{c}\mathrm{C} 1: \\
\mathrm{I}=2\end{array}$ & $\begin{array}{c}\mathrm{C} 1: \\
\mathrm{F}=2\end{array}$ & $\begin{array}{c}\mathrm{C} 2: \\
\mathrm{I}=2\end{array}$ & $\begin{array}{c}\mathrm{C} 2: \\
\mathrm{n}=2\end{array}$ & $\begin{array}{c}\mathrm{C} 3: \\
\mathrm{n}=2\end{array}$ & Sum \\
\hline $\begin{array}{c}\text { Error } \\
\text { framing }\end{array}$ & 1 & $2^{*}$ & 0 & $2^{*}$ & 2 & $1^{*}$ & 8 \\
\hline $\begin{array}{c}\text { Other } \\
\text { students }\end{array}$ & 1 & 2 & 2 & 0 & 1 & 2 & 8 \\
\hline Building & 0 & 0 & 2 & 2 & 1 & 1 & 6 \\
\hline Neutral & 0 & 2 & 0 & 0 & 1 & 2 & 5 \\
\hline Names & 0 & 1 & 0 & 1 & 1 & 1 & 4 \\
\hline Groups & 1 & 1 & 0 & 1 & 1 & 0 & 4 \\
\hline $\begin{array}{l}\text { Thinking } \\
\text { Time }\end{array}$ & 0 & 0 & 1 & 1 & 0 & 0 & 2 \\
\hline \hline
\end{tabular}

*These students did not give specific error framing-type

statements, but their responses suggest an error-friendly climate.

\section{A. Error framing}

In their training, GTAs were prompted to practice error framing with avatar-students in the simulator and were observed to use it in their classrooms. Notably, three students perceived their anxiety with cold calling to decrease when their GTA used an error framing-type statement in response to student answers. Brocky (P1) [25] explained that they were nervous with cold calling at first but their GTA's response to an incorrect answer helped them feel more comfortable. They provided an example:

Int.: Do you think that's typical of your physics lab with professors like that or is it kind of that specific GTA has a really good way of handling wrong answer?

Brocky: Yeah. I think it's more specifically him 'cause he knows, he'll [P1] say 'I know this is really hard so don't worry if you answer it wrong'.

Brocky perceived that GTA's response mitigated their nervousness with cold calling as their GTA emphasized effort over correctness. While three students gave specific examples of GTA responses similar to Brocky's example, five other students reported an overall feeling of not being afraid to share an incorrect answer in front of the class. Amari (C2) and Jamie (C2) explained their reasoning:

Amari: Ifelt like it made me more comfortable with doing something wrong because... 
Jamie: I think he was very big on the fact that we were just learning this stuff and it wasn't about getting the right answers, but like the process.

Val (C1) shared similar feelings about their GTA's responses to incorrect student answers:

Int.: ... when you don't necessarily know the right answer when you're called on, do you feel okay with giving a response...?

Val: ... he makes it known that you don't need to have a correct answer. Any answer is a good answer as long as you are participating...

In total, eight students reported GTA responses that were either consistent with the definition of error framing or with the implication of an error-friendly climate existed in their classroom.

\section{B. Allowing other students to assist}

Eight students expressed comfort with cold calling when their GTA gave them the option to say, "I don't know" and allowed their peers to help with the cold-call question. When asked about how being called on affected their feelings of anxiousness, Marley (C3) responded:

Marley: I mean I didn't really care ... 'cause I feel like sometimes when like you're called on and you have to give an answer, that's where the anxiety comes from but there are people who are like 'I have no idea' and he was like 'okay' and he picked someone else so.

Bo (C1) and Jordan (C1) described their reasoning about how their GTA allowing others to help them answer the cold call helped reduce their anxiety:

Int: ... why do you feel like the pressure was relieved?

Bo: Because not only is he looking for the answer, but there's also other people in the class that are looking for the answer.

Jordan: I feel like it saves time. If I'm dead in my tracks and have to sit there for a good minute while everyone looks at me waiting for an answer, oh man is that uncomfortable!

These examples are representative of the ways students described GTAs allowing them to back out of a cold call decreased their feelings of anxiousness associated with cold calling.

\section{Building off what students know}

Six students perceived their GTA to build off their prior knowledge by encouraging students to say what they did know or by asking questions about content students already knew during cold calling. Amari (C2) was asked to explain:

Int.: So, you say he was nice about it (cold calling), could you elaborate more on that?

Amari: So, if he called on you and you didn't know, he would encourage you to say something that you did know, and then he would build off of that.

Like Amari's GTA, the other five students reported their GTA to encourage them to say what they already knew as a way of alleviating their feelings of anxiousness with cold calling.

\section{Providing neutral responses with explanations}

Five students felt relieved after being cold called when their GTA responded the same way regardless of the correctness of a student's answer. Marley (C3) described similar feelings as the other four students. When asked what their GTA had done to make them feel comfortable with sharing their ideas, Marley responded:

Marley: I think that whether you were right or wrong like there was always an explanation. So, I mean, it's like when I was wrong like you didn't feel like it.

Marley felt comfortable with sharing their ideas because their GTA would respond in a similar way to any student response with an explanation.

\section{E. Using student names}

Four students expressed they felt more comfortable with being called on when GTAs learned their names and used them as part of the cold call. Val (C1) was the only one to explain their reasoning:

Int.: ... having him know your name and knowing that you could possibly be called on, does that affect your feelings in the classroom?

Val: I feel like yeah because he knows my name, because it's more like I know everybody in the class, it's such a small class, I feel better about answering and being called on.

Val explained how their GTA knowing student names made them feel connected to their classmates and more comfortable sharing their answer in front of the class. Learning student names has been suggested to be the easiest way for an instructor to demonstrate student care [26]. Undergraduate biology students expressed similar feelings to Val when the bridge program faculty used their names when interacting with them resulting in the student perception of the faculty to be approachable and helpful [27].

\section{F. Calling on student groups}

Four students perceived their GTA to cold call on student groups during whole class discussions. Quinn (C1) expressed why they felt comfortable sharing their ideas:

Quinn: ... within our groups we have multiple brains, and we were all thinking together, and then we would share as a group. So that's good at making people comfortable because it's not just yourself talking.

Quinn felt comfortable responding to a cold call when they were able to respond as a group because they were not alone. Brocky (P1) shared an example from their class:

Brocky: ...before he's gone around the room and every group will, like one person will answer what they think and then we'll just compare... which is nice 'cause everyone does have a different answer, so it's not like one person's wrong. 
Int: Okay. How does that way of doing it impact your feeling of anxiousness?

Brocky: A lot less.

Similar to the other two students, Quinn and Brocky felt comfortable with cold calling when their GTA called on their student group because they were not alone with responding. Further investigation is necessary about the impact of calling on student groups on student anxiety because students have reported a mixture of negative and positive impacts on their anxiety when working in groups [11].

\section{G. Providing thinking time before cold calling}

Two students perceived their GTA to provide thinking time before they called on students or expected a student response. Bo $(\mathrm{C} 1)$ explained:

Bo: ...he'd ask you a question, and we would've already written it down. So, I don't really have to worry about what I'm going to say because I've already written it down, so I would just reciprocate what I wrote down but in a shortened, more concise manner.

As Bo explained, time to think before answering mediated the pressure of thinking in the moment. Similarly, Cooper, Downing and Brownell found students reported their anxiety to increase with cold calling when they were not given enough time to articulate their thoughts before they were expected to respond [11].

\section{DISCUSSION AND IMPLICATIONS}

In this study, we found students perceived their GTA to implement a variety of teaching strategies that reduced their anxiety associated with cold calling. Prior research has similarly shown students enrolled in active learning biology courses in community college expressed they would feel more comfortable with cold calling if their instructor paired it with other teaching strategies [28]. These findings support our hypothesis that pairing cold calling with another teaching strategy can reduce student anxiety associated with cold calling.

Our findings also indicate that GTAs might already be aware of the anxiety associated with cold calling and are proactive about mitigating student anxiety with other teaching strategies. However, different students may have different reactions to the same strategy even when used by the same GTA. Therefore, we recommend that GTA preparation include rehearsal with a variety of teaching strategies that increase student participation and reduce student anxiety, as it is not likely that one strategy will work for all students. As the literature about how an instructor can effectively reduce student anxiety with cold calling is limited, future research could include investigating how GTAs implement cold calling in their courses and which teaching strategies implemented by GTAs are the most effective for minimizing student anxiety with cold calling.

In addition, eight of the eleven students described cold calling paired with an error framing statement or error- friendly climate to reduce their anxiety associated with cold calling, similar to what community college biology students suggested might reduce their anxiety associated with cold calling [28]. Error framing was a teaching strategy the participating GTAs had practiced pairing with cold calling at the beginning of the semester. Future research could investigate how error framing paired with cold calling can mitigate the anxiety associated with cold calling. Furthermore, we are currently comparing GTA classroom observation data between semesters with and without the simulator training to investigate if the intervention influenced the GTAs to choose error framing as a teaching strategy. The results could inform future GTA training.

Nine out of eleven students described the actions of their GTA to be relatable, personable, and helpful, which created a welcoming environment for them to share their ideas. The student perception of their GTA creating a welcoming environment is similar to a strategy described by KerssenGriep as instructor "communication maintaining a climate safe for independent thought and risk-taking" specifically when instructors learned student names and shared personal information [29]. During analysis we identified that studentperceived teaching strategies, like using student names and providing thinking time, aligned with core constructs of the conceptual framework facework (actions taken to prevent threats to and to maintain students' self-image) [29-32]. Instructor use of facework has been linked to an increase in students' willingness to participate in classroom activities and with students' course satisfaction [31,32]. Future investigations about the impact of teaching strategies on student affective behaviors, like anxiety, could be guided by such a framework that has been applied in other areas of education research.

\section{LIMITATIONS}

The generalizability of our claims are limited by several factors. Our sample size was small, and we did not investigate our findings across identity groups. Moreover, it is possible the interviews filtered students who do not have high levels of social anxiety in their classroom.

We only investigated one instructional setting. Here, we focused on small sections (24-32 students); however the class size and type of instructor (i.e., faculty or GTA) could have influenced the anxiety with cold calling. Cooper et al. also found students expressed more comfort with cold calling in a smaller class [11]. It is difficult to disentangle the teaching strategies with the size of the classroom, as seen in Val's quote about their GTA's use of student names. Additionally, students' comfort with cold calling may vary across instructor type. Future research could compare how different instructional styles impact student anxiety.

\section{ACKNOWLEDGMENTS}

This work is supported in part by the National Science Foundation DUE Grant No. 1725554. 
[1] E. A. Becker, et al., The effects of practice-based training on graduate teaching assistants' classroom practices. CBE Life Sci. Educ., 16 (4), ar58 (2017).

[2] G. Harris, J. Froman, and J. Surles, The professional development of graduate mathematics teaching assistants. Int. J. Math. Educ. Sci. Technol., 40(1), 157-172 (2009).

[3] L. B. Wheeler, J. L. Maeng, and B. A. Whitworth, Teaching assistants' perceptions of a training to support an inquiry-based general chemistry laboratory course. Chem. Educ. Res. Pract., 16(4), 824-842 (2015).

[4] T. D. Reeves, T. D., L. E. Hake, X. Chen, J. Frederick, K. Rudenga, L. H. Ludlow, and C. M. O'Connor, Does context matter? Convergent and divergent findings in the crossinstitutional evaluation of graduate teaching assistant professional development programs. CBE Life Sci. Educ., 17(1), ar8 (2018).

[5] T. D. Reeves, G. Marbach-Ad, K. R. Miller, J. Ridgway, G. E. Gardner, E. E. Schussler, and E. W. Wischusen, A conceptual framework for graduate teaching assistant professional development evaluation and research. $\underline{\mathrm{CBE}}$ Life Sci. Educ., 15(2), es2 (2016).

[6] K. D. Tanner, Structure matters: twenty-one teaching strategies to promote student engagement and cultivate classroom equity. CBE-Life Sci. Educ., 12(3), 322-331 (2013).

[7] D. Lemov, (2010). Teach like a champion: 49 techniques that put students on the path to college $(K-12)$ (John Wiley \& Sons, San Francisco, 2010), Vol. 1, p. 111-114, 221-223.

[8] E. J. Dallimore, J. H. Hertenstein, M. B. Platt, Impact of coldcalling on student voluntary participation. J. Manag. Educ., 37(3), 305-341 (2013).

[9] S. L. Eddy, S. E. Brownell, and M. P. Wenderoth, Gender gaps in achievement and participation in multiple introductory biology classrooms. CBE Life Sci. Educ., 13(3), 478-492 (2014).

[10] B. J. England, J. R. Brigati, and E. E. Schussler, Student anxiety in introductory biology classrooms: Perceptions about active learning and persistence in the major. PloS one, 12(8), e0182506 (2017).

[11] K. M. Cooper, V. R. Downing, and S. E. Brownell, The influence of active learning practices on student anxiety in large-enrollment college science classrooms. Int. J. STEM Educ., 5(1), 23 (2018).

[12] D. Watson and R. Friend, Measurement of social-evaluative anxiety. J. Consult. Clin. Psychol., 33(4), 448 (1969).

[13] S. L. Eddy, M. Converse, and M. P. Wenderoth, PORTAAL: a classroom observation tool assessing evidence-based teaching practices for active learning in large science, technology, engineering, and mathematics classes. CBE Life Sci. Educ., 14(2), ar23 (2015).

[14] N. Keith and M. Frese, Effectiveness of error management training: A meta-analysis. J. Appl. Psychol., 93(1), 59-69 (2008).

[15] B. S. Bell and S. W. Kozlowski, Active learning: effects of core training design elements on self-regulatory processes, learning, and adaptability. J. Appl. Psychol., 93(2), 296 (2008).

[16] M. Kapur and K Bielaczyc, Designing for productive failure. Journal of the Learning Sciences, 21(1), 45-83 (2012).
[17] R. W. Bybee and N. M. Landes, Science for Life \& Living: An Elementary School Science Program from Biological Sciences Curriculum Study. Am. Biol. Teach. 52, 92 (1990).

[18] M. Wilcox, Y. Yang, and J. J. Chini, Quicker method for assessing influences on teaching assistant buy-in and practices in reformed courses. Phys. Rev. Phys. Educ. Res. 12, 020123 (2016).

[19] J. J. Chini, C. L. Straub, C. L., and K. H. Thomas, Learning from avatars: Learning assistants practice physics pedagogy in a classroom simulator. Phys. Rev. Phys. Ed. Res., 12(1), 010117 (2016).

[20] J. B. Velasco, A. Knedeisen, D. Xue, T. L. Vickrey, M. Abebe, and M. Stains, Characterizing instructional practices in the laboratory: The Laboratory Observation Protocol for Undergraduate STEM. J. Chem. Educ., 93(7), 1191-1203 (2016).

[21] Students were prompted to talk about their feelings of anxiousness associated with cold calling when (1) they anticipated being called on and (2) they are being called on.

[22] D. M. Fetterman et al., in Qualitative approaches to evaluation in education: The silent scientific revolution, edited by D. M. Fetterman (Praeger Publishers, 1988), Vol. 1, p. 212 213.

[23] K. Gwet, Kappa statistic is not satisfactory for assessing the extent of agreement between raters. Statistical Methods for Inter-Rater Reliability Assessment Series 2, 9 (2002).

[24] K. L. Gwet, Handbook of Inter-Rater Reliability: The Definitive Guide to Measuring the Extent of Agreement Among Raters 4th ed. (Advanced Analytics, Gaithersburg, 2014), Vo1. 1 p. $104-107$.

[25] We did not collect demographic information (e.g., gender, race/ethnicity) from the interview participants. Thus, we will refer to all participants with gender neutral pronouns (i.e., they/them/their) and with gender neutral pseudonyms. We recognize the exclusion of demographic information for students and GTAs is a limitation to this study.

[26] J. D. H. Gaffney and J. T. Whitaker, Making the Most of Your First Day. Phys. Teach., 53(3), 137 (2015).

[27] K. M. Cooper, M. Ashley, and S. E. Brownell, Breaking Down Barriers: A Bridge Program Helps First-Year Biology Students Connect With Faculty. J. Coll. Sci. Teach., 47(4), 60-70 (2018).

[28] V. R. Downing et al., Anxiety in community college active learning courses. CBE Life Sci. Educ., (to be published).

[29] J. Kerssen-Griep, Teacher communication activities relevant to student motivation: Classroom facework and instructional communication competence. Commun. Educ., 50 (3), 256-273 (2001).

[30] E. Goffman, On face-work: An analysis of ritual elements in social interaction. Psychiatry, 18(3), 213-231 (1955).

[31] J. Kerssen-Griep, J. A. Hess and A. R. Trees, Sustaining the desire to learn: Dimensions of perceived instructional facework related to student involvement and motivation to learn. Western J. Comm., 67(4), 357-381 (2003).

[32] J. D. Gaffney and A. L. H. Gaffney, Student satisfaction in interactive engagement-based physics classes. Phys. Rev. Phys. Ed. Res., 12(2), 020125 (2016). 